\title{
Reliability of arterial stiffness indices at rest and following a single- bout of moderate-intensity exercise in older adults.
}

\author{
MARIA PERISSIOU ${ }^{1}$ \\ TOM G. BAILEY ${ }^{1}$ \\ MARK WINDSOR $^{1}$ \\ ANTHONY S. LEICHT ${ }^{2}$ \\ JONATHAN GOLLEDGE ${ }^{3,4}$ \\ CHRISTOPHER D. ASKEW ${ }^{1}$ \\ ${ }^{1}$ VasoActive Research Group, School of Health and Sport Sciences, University of the Sunshine \\ Coast, Queensland, Australia. \\ ${ }^{2}$ Sport and Exercise Science, James Cook University, Townsville, Queensland, Australia. \\ ${ }^{3}$ Queensland Research Centre for Peripheral Vascular Disease, James Cook University, \\ Townsville, Queensland, Australia. \\ ${ }^{4}$ Department of Vascular and Endovascular Surgery, The Townsville Hospital, Townsville, \\ Queensland, Australia.
}

RUNNING TITLE: Reliability of resting and post-exercise arterial stiffness

WORD COUNT: 3,767

FIGURES: 1 TABLES: 3

\section{AUTHOR FOR CORRESPONDENCE:}

A/Prof Christopher Askew. VasoActive Research Group - School of Health and Sport Sciences,

University of the Sunshine Coast, Locked bag 4, Maroochydore DC, Australia. Email: caskew@usc.edu.au; Phone+61 754565961 


\section{Summary}

Short-term changes in arterial stiffness with exercise are proposed to better reflect vascular impairments than resting measures alone, and are suggested as a prognostic indicator of cardiovascular risk in older adults. Arterial stiffness indices are reliable at rest, but the time-course and reliability of post-exercise changes in arterial stiffness in older adults is unknown. The precision of post-exercise changes in arterial stiffness should be determined prior to their use in large prospective trials. This study assessed the between-day reliability of the changes in pulse wave velocity (PWV), augmentation index (AIx75) and reflection magnitude (RM) following an exercise bout in older adults. Ten older adults $(71 \pm 5 \mathrm{y})$ were tested on three separate days, 7 days apart. PWV, AIx 75 and RM were assessed at rest, immediately post, and at 20, 40 and 60 min during recovery after moderate-intensity cycling. Intraclass correlation coefficient (ICC) and reliability coefficient (RC) were used to assess the relative and absolute reliability of arterial stiffness responses. PWV increased, and RM decreased immediately after exercise $(\mathrm{P}<0.05)$, and returned to baseline during recovery. AIx decreased during recovery $(\mathrm{P}<0.001)$. Resting ICC values were 0.91(PWV), 0.72 (AIx75) and 0.40 (RM). Reliability of the immediate changes following exercise was high for PWV (ICC:0.87, RC:1.9 m.s $\mathrm{s}^{-1}$ ), and moderate for AIx75(ICC:0.64, RC:7\%) and RM(ICC:0.59, RC:9\%). Reliability of the post-exercise responses was similar to that at rest for all measures of arterial stiffness. These findings indicate that post-exercise changes in arterial stiffness indices are reliable in healthy older adults, and supports further investigation of the prognostic value of these responses.

KEY WORDS: Pulse wave velocity, augmentation index, reflection magnitude, blood pressure, cardiovascular risk, ageing. 


\section{INTRODUCTION}

Arterial stiffness increases with age (McEniery et al. 2005) and is one of the earliest detectable manifestations of adverse structural and functional vascular changes, contributing to the pathogenesis of cardiovascular disease (Steppan et al. 2011) and end-organ damage (Lee et al. 2010). Carotid to femoral pulse wave velocity (PWV) is the criterion measure for the non-invasive assessment of central aortic arterial stiffness (Cecelja et al. 2012), the region which exhibits the greatest age-related stiffening (Mitchell et al. 2004, Wilkinson et al. 2010, Lee Stoner 2012). Augmentation index (AIx) provides an integrated summary of wave reflection timing and amplitude (Nichols et al. 2002), defined as the central augmentation pressure as a percentage of pulse pressure. Wave separation analysis enables the calculation of reflection magnitude (RM) expressed as the ratio of reflected (backward, $\mathrm{Pb}$ ) to forward (Pf) pressure waves (Westerhof et al. 2006). While resting indices of arterial stiffness are used to determine the risk of cardiovascular disease development and mortality (Mitchell et al. 2010, Chirinos et al. 2012), the augmented haemodynamic changes with exercise may be more sensitive for revealing vascular abnormalities and cardiovascular risk in healthy adults.

Arterial stiffness increases immediately after exercise before returning to, or below, resting levels during a period of recovery in healthy, younger adults (Mutter et al. 2017). However, the increase in arterial stiffness after exercise has been shown to be exaggerated, and remain above baseline levels during recovery in adults with known cardiovascular risk factors, such as obese individuals (Shim et al. 2011, Bunsawat et al. 2017) and adults with untreated hypertension (Gkaliagkousi et al. 2014). Increases in sympathetic nerve activity (Smith et al. 2015), oxidative stress (Kawamoto et al. 2016) and endothelial dysfunction (Duprez 2010) following exercise are implicated in arterial stiffening, and it is also these underlying mechanisms that contribute to the long-term development of cardiovascular disease and mortality (Dhalla et al. 2000, Widmer et al. 2014, La Rovere et al. 2015). Sustained elevations in arterial stiffness expose the brain and kidneys to high pulsatile central blood pressure and microcirculatory dysfunction (Lee and Oh 2010), which are linked to future risk of stroke and hypertension. For example, an increase in PWV after exercise was strongly associated 
with abnormal markers of kidney function in older adults (Keith et al. 2013), and therefore monitoring changes in arterial stiffness and central blood pressure during the short-term recovery period after exercise may provide important prognostic information and assist to identify at-risk older adults.

Using non-invasive oscillometric assessment (SphygmoCor Xcel; AtCor Medical, Sydney Australia), the reliability of resting PWV and AIx75 is well established in middle- and older-aged adults (ICC 0.75-0.99) (Hwang et al. 2014, Mitchelmore et al. 2017). Exercise may provide a greater signal to noise ratio for improving the sensitivity of arterial stiffness measures, and reports of reliable changes in PWV (ICC: 0.94) (Keith et al. 2013) and AIx75 ( ICC: 0.82) (Lim et al. 2016) in response to exercise in younger adults support the use of this approach. There remains a need to determine the test reliability (precision) across repeated assessments in older adults, who would benefit the most from accurate CVD risk prognosis. This would enable the smallest detectable changes in arterial stiffness with exercise to be established, and therefore support the potential use of these measures for the assessment of cardiovascular risk and prognosis. Therefore, by conducting three repeated assessments that were each separated by one week, this study aimed to determine the reliability of PWV, AIx 75, and RM at rest, immediately after, and during post-exercise recovery following a bout of moderate-intensity cycling exercise in older adults.

\section{MATERIALS AND METHODS}

\section{Participants}

Ten healthy older participants ( $71 \pm 5$ years) were recruited through local community announcements and a University alumni group. Participants were included if they were aged 60-86 years, nonsmokers, $\mathrm{BMI}<39$, and had no musculoskeletal problems that limited exercise participation. Participants were excluded if they had a history of cardiovascular, metabolic or renal disease. Participants with medically untreated hypertension (defined as an average SBP $\geq 140 \mathrm{mmHg}$ and/or an 
average DBP $\geq 90 \mathrm{mmHg}$ ) were also excluded. All participants were fully informed of the study procedures and provided written informed consent prior to participation. The study conformed to the Declaration of Helsinki (1964) and was approved by the institutional ethics committees.

\section{General experimental procedures}

Participants visited the exercise physiology laboratory at the University of the Sunshine Coast on 4 occasions, each separated by at least 7 days. During the first visit, anthropometric data were collected before undertaking a maximal incremental cycling test for the determination of peak power output (PPO) and peak cardiorespiratory fitness $\left(\dot{V} O_{2 p e a k}\right)$. Each participant then underwent three repeat experimental visits at the same time of the day in order to reduce the potential confounding influence of diurnal variation on cardiovascular function (Dhaun et al. 2014). Each experimental visit (visits 24) consisted of a bout of moderate-intensity cycling exercise. Measures of arterial stiffness (PWV, RM, AIx) were made while participants were supine; 1) at rest before exercise, 2) immediately after exercise (0-5 min), and 3) during 60 min of recovery. Prior to attending the laboratory on each occasion, participants were instructed to abstain from exercise for $24 \mathrm{~h}$, caffeine and alcohol for $12 \mathrm{~h}$, and to be fasted for $3 \mathrm{~h}$.

Maximal incremental cycling test : An incremental cycling test was conducted to determine peak oxygen uptake ( $\left.\dot{V} O_{2 p e a k}\right)$. The test was performed on an upright cycle ergometer (Lode Corival, Lode B.V., Groningen, Netherlands) until volitional exhaustion (McDermott et al. 1998). Oxygen uptake was measured using a Parvo Medics TrueOne 2400 metabolic cart and software (Parvo Medics, East Sandy UT, USA). Following a 3-min warm up at $0 \mathrm{~W}$, the test commenced at $20 \mathrm{~W}$ and increased by $10 \mathrm{~W}$ per min until volitional exhaustion. Heart rate (HR) via 12-lead ECG (Mortara Inc., WI, USA) and rating of perceived exertion (RPE), using the 0-10 Borg scale (Pfeiffer et al. 2002), were measured every 60 s during the test. Maximal effort during the test was confirmed based on the presence of the following: heart rate $>90 \%$ of age-predicted maximum; respiratory exchange ratio $>1.15$; $\mathrm{RPE}>9$; a sustained fall in pedal cadence $(>10$ revolutions per minute, $\mathrm{RPM}) . V \mathrm{O}_{2 \text { peak }}$ 
was determined as the highest $15 \mathrm{~s}$ average over the last 60 s of peak exercise. From this, peak power output (PPO) at $V \mathrm{O}_{2 \text { peak }}$ was used to calculate the workload for the moderate-intensity exercise bout in the experimental visits (Ferguson 2014).

Moderate-intensity continuous exercise (visits 2-4): Participants performed a bout of cycling exercise consisting of a 3-min standardised warm-up at $0 \mathrm{~W}$, followed by 24 min of moderate-intensity continuous cycling (40\% PPO) at a cadence of 60 RPM (Lode Corival, Lode B.V., Groningen, Netherlands). This protocol was designed to align with current exercise session recommendations for older adults (20 to $30 \mathrm{~min}$ at 40 to $60 \%$ of heart rate reserve or 40 to $60 \%$ PPO)(Garber et al. 2011). During exercise, HR (using 12-lead ECG) and RPE were measured every 60s. Brachial blood pressure was measured every 6-min using a manual sphygmomanometer.

Arterial stiffness measurements: Indices of arterial stiffness (PWV, RM and AIx) were measured at baseline after $15 \mathrm{~min}$ of quiet rest in the supine position, using the SphygmoCor XCEL device (AtCor Medical, West Ryde, NSW, Australia). Measurements were repeated immediately following exercise (0-5 min) and at 20, 40 and 60 min during exercise recovery. Participants were instructed to remain quiet and still for $5 \mathrm{~min}$ before and during each arterial stiffness measurement. The measurement of wave reflection characteristics (AIx and RM) preceded PWV at all time-points, and a complete cycle for all arterial stiffness measurements took $\sim 5 \mathrm{~min}$.

Wave reflection characteristics: Brachial artery waveforms were recorded on the right upper arm, following manufacturer guidelines (Hwang et al. 2014) by inflating a brachial cuff to supradiastolic pressure, approximately midway between the shoulder and the elbow. Each measurement cycle commenced with three repeat measurements of brachial blood pressure, and an average of the last two measurements were used. Subsequently, a corresponding aortic pressure waveform was generated by applying proprietary digital signal processing and transfer function (Butlin et al. 2012), from which central systolic (cSBP), diastolic (cSDP), pulse pressure (cPP), augmentation pressure (AP), AIx, RM, Pf and $\mathrm{Pb}$ were derived. Central pulse pressure was calculated as the difference 
between cSBP and cDBP. Augmentation pressure is defined as the difference between cSBP and the pressure at the inflection point caused by the merging of forward and reflected pulse wave. AIx was defined as the augmentation pressure expressed as a percentage of pulse pressure. As AIx is significantly affected by heart rate, the index was corrected for a heart rate at 75 beats per minute (AIx75). Wave separation analysis was applied using SphygmoCor CVMS software (AtCor Medical, Sydney, Australia). This method creates an assumed triangular-shaped flow wave by aligning the start, peak, and end of the flow wave with the foot, inflection point, and notch of the aortic pressure wave, respectively (Westerhof et al. 2006). Based on the assumed flow wave, the aortic forward (Pf) and backward $(\mathrm{Pb})$ pressure waveforms were calculated. Reflection magnitude $(\mathrm{RM})$ was calculated as the ratio of $\mathrm{Pb}$ to $\mathrm{Pf}$ and expressed as a percentage: $\mathrm{RM}=\mathrm{Pb} / \mathrm{Pf} * 100$.

Pulse wave velocity (PWV): To assess carotid-femoral PWV, carotid pulse waves were obtained by applanation tonometry of the right carotid artery, and femoral pulse waves were obtained by sphygmomanometry of the right thigh using a thigh cuff. The tonometer was positioned at the site of the carotid pulse whilst a femoral cuff was placed at mid-thigh. Following the operator's guidelines (SphygmoCor Xcel V1), the distance between the carotid and femoral arteries was measured from the carotid site above the suprasternal notch to the proximal edge of a thigh cuff over the femoral artery at the leg midway between the hip and the knee. The distance from the inguinal fold to the femoral cuff was standardised at $200 \mathrm{~mm}$. This distance and the same placement of the thigh cuff was then used for all repeat sessions for each individual. In the supine position, participants were asked to breathe steadily and remain relaxed to facilitate an optimal carotid pulse tonometry measurement. Once a regular carotid pulse was detected, femoral pulse waves were collected simultaneously by partially inflating the thigh cuff to $80 \mathrm{mmHg}$. PWV was then determined by calculating the ratio of the distance between the pulse measuring sites to the time delay between the carotid and femoral pulse waves (Wilkinson et al. 2010). 


\section{Statistical Analyses:}

Arterial stiffness indices (PWV, AIx75 and RM) were measured at 1) rest (pre-exercise), 2) immediately post-exercise ( $0 \mathrm{~min}$ post), and 3 ) during 0-60 min of post-exercise recovery, on three separate occasions. Using the trapezoidal rule (Tallarida and Murray 1987), total area under the curve (AUC) of arterial stiffness indices (0-60 min post-exercise) was calculated to quantify total recovery. Data were also calculated as changes from rest (delta) to account for the small, but non-significant day-to-day variance in resting arterial stiffness values. All data were initially tested for normality and screened for outliers. To determine whether there were any significant changes in arterial stiffness indices at rest or following exercise between the three visits we initially included all data in a twoway (visit*time) linear mixed model (LMM). A two-way (visit*time) LMM was also used to detect differences in post-exercise delta measures of arterial stiffness between visits. All analyses were performed using SPSS (version 21; SPSS, Chicago, IL, USA). All data are presented as mean (95\% confidence interval; 95\% CI), unless otherwise specified, and statistical significance was set at $\mathrm{P}<0.05$. For $\mathrm{P}$ values $<0.000$, the value is reported as $\mathrm{P}<0.001$.

The reliability of arterial stiffness indices (PWV, AIx75 and RM) at 1) rest, 2) immediately postexercise, and 3) the recovery AUC, across the three repeated visits, was initially determined comparing the mean differences between the three visits using a one-way LMM, and was further characterised using the intraclass correlation coefficient (ICC), the coefficient of variation (ratio of standard deviation to the mean, $\mathrm{CV} \%$ ), standard error of the mean (SEM), and the reliability coefficient (RC) (Weir 2005). ICC was used to assess the relative reliability of arterial stiffness indices, as ICC accounts for both the consistency of arterial stiffness from test to re-test (withinparticipant change), as well as the systemic change in the mean between visits. The ICC was calculated as the ratio of the squared between subject variance to the sum of squares of between and within subject variance $\left[\mathrm{SD}_{\mathrm{b}}{ }^{2} /\left(\mathrm{SD}_{\mathrm{b}}{ }^{2}+\mathrm{SD}_{\mathrm{w}}{ }^{2}\right)\right]$. ICC values above 0.75 were considered to indicate excellent reliability, $0.40-0.74$ good reliability, and less than 0.40 suggests poor reliability (Fleiss 1999). The RC was used as a measure of absolute reliability, which accounts for variability in arterial 
stiffness due to random and systemic measurement error. The RC quantifies absolute reliability measurement error in the same units as the measurement itself. RC was calculated by multiplying the within-participant SEM by 2.77 ( $\sqrt{ } 2 * 1.96)$ (Weir 2005, Vaz et al. 2013).

\section{Results}

\section{Participant Characteristics}

Participant characteristics are presented in Table $1 . \dot{V} O_{2 p e a k}$ was $24.6 \mathrm{ml} \cdot \mathrm{kg}^{-1} \cdot \mathrm{min}^{-1}$ (95\% CI 18.9 to 30.3), which can be characterised as fair according to normative age- and sex-specific data (Garber et al. 2011).

\section{Exercise variables}

Heart rate increased by $21 \mathrm{bpm}(95 \% \mathrm{CI} 18$ to $29, \mathrm{P}=0.003)$ during cycling exercise and did not differ between visits $(\mathrm{P}=0.465)$. Similarly, MAP increased by $8 \mathrm{mmHg}(95 \% \mathrm{CI} 4$ to $10, \mathrm{P}=0.002)$ during cycling exercise, with no differences observed between visits $(\mathrm{P}=0.840)$. $\mathrm{RPE}$ increased by $2(95 \% \mathrm{CI}$ 1 to $3, \mathrm{P}<0.001)$ during exercise, and did not differ between visits $(\mathrm{P}=0.431)$.

\section{Resting and post-exercise measures of arterial stiffness}

Figure 1 shows the mean responses for PWV (Fig 1A), AIx75 (Fig 1B) and RM (Fig 1C) at rest and at each time point after exercise for the three repeat visits. There was no difference in resting values of arterial stiffness indices between visits. PWV increased from baseline to immediately after exercise

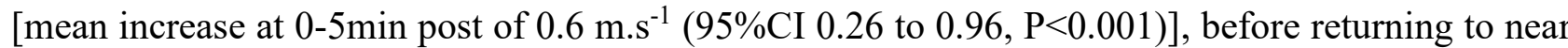
baseline levels. The increase in AIx75 was negligible immediately after exercise, before decreasing below baseline levels at 40 and 60 min post-exercise (60 min decrease of $3.5 \%$ (95\%CI 1.4 to 5.7 , $\mathrm{P}<0.001)$. RM decreased immediately after exercise [mean decrease at $0-5$ min post of $10.1 \%$ (95\%CI 5.3 to $14.8, \mathrm{P}<0.001)]$ before returning to near baseline levels. 
A main effect for "visit" was observed for PWV (Fig 1A), where mean PWV (across all time points) during visit 3 was lower compared to visit $1(\mathrm{P}<0.001)$ and visit $2(\mathrm{P}=0.042)$. Conversely, AIx 75 (Fig 1B) was higher during visit 1 compared to visit $2(\mathrm{P}=0.006)$, but not compared to visit $3(\mathrm{P}=0.084)$. There were no significant visit*time interactions for PWV, AIx75, and RM (Fig 1A-C), indicating that the response to exercise (time effect) was consistent across visits. This was confirmed when the change in each variable from baseline (delta) was assessed, which showed that there was no difference in the arterial stiffness response across the visits (Table 2).

\section{Reliability of arterial stiffness at rest, immediately post-exercise, and during recovery}

Measures of reliability, and the corresponding mean data, for the indices of arterial stiffness at rest, immediately post-exercise, and during recovery, across the three visits are shown in Table 3. Reliability of PWV at rest was excellent (ICC $>$.75), and more reliable than the other resting measures of vascular stiffness, particularly RM for which the reliability was borderline good-poor (ICC: 0.40 ). Reliability of the post-exercise measures of vascular stiffness was similar to that observed at rest for PWV and AIx; whereas reliability was slightly improved for RM with an increase in the ICC (0.40 to 0.59 ) and a reduction in the reliability coefficient (11 to $9 \%$ ) post-exercise compared with rest. Recovery of each vascular stiffness measure during the 60-min period after exercise (area under the curve) did not change across the three visits, and the reliability of these recovery measures was similar to that observed at rest, as indicated by the comparable ICC and CV\% values (Table 3).

\section{Discussion}

The present study examined the between-day reliability of exercise-induced, short-term changes in arterial stiffness in healthy older adults. Our main findings were that carotid-femoral PWV and AIx75 can be measured reliably in healthy older individuals at rest, immediately after, and during one hour of recovery from moderate-intensity cycling exercise across three separate occasions. Whilst, RM demonstrated poor reliability at rest, we demonstrate that this index can be measured reliably immediately after and during recovery from exercise. 


\section{Reliability of resting arterial stiffness indices}

We show that the between-day relative reliability of resting carotid-femoral PWV was excellent in older adults (ICC: 0.91), consistent with a recent study using the same device in young individuals (ICC: 0.98) (Hwang et al. 2014). Furthermore, the ICC values (0.72-0.57) for AIx75, Pf and Pb at rest were consistent with recent studies (ICC, AIx75:0.70-0.75; Pf: 0.66; Pb: 0.76) in young and older individuals (Stoner et al. 2016, Mitchelmore et al. 2017). Despite good reliability of the forward and backward pressure wave, the reliability of $\mathrm{RM}$ at rest was poor, as also observed previously (Stoner et al. 2016). RM is an index that may be sensitive to small changes (Weir 2005), and vulnerable to signal noise (variance) compared with PWV and AIx75. The excellent relative reliability of PWV at rest corresponded with an absolute reliability (RC: $1.9 \mathrm{~m} . \mathrm{s}^{-1}$ ) similar to that reported by Keith et al. (2013) $\left(1.5 \mathrm{~m} . \mathrm{s}^{-1}\right)$. While the clinical meaningfulness of this smallest detectable change remains to be fully established in older adults, it has previously been shown that a $1.0 \mathrm{~m} \cdot \mathrm{s}^{-1}$ (or 1SD) increase in PWV is associated with a 10\% increase in cardiovascular risk (Vlachopoulos et al. 2010).

Time-course of the changes in arterial stiffness after exercise.

PWV increased immediately after exercise, followed by a transient return to baseline. We also observed a transient decrease in AIx75 and reflection magnitude to below resting levels after exercise. These observations are the first in older individuals and are similar to the responses recently reviewed in healthy younger adults (Mutter et al. 2017). While resting measures of arterial stiffness reflect structural and functional mechanisms, beneficial reductions in arterial stiffness during recovery following acute exercise in healthy adults are suggested to be functional and are likely mediated by reductions in central blood pressure (Millen et al. 2016), vasoconstrictors (endothelin-1) (Schreuder et al. 2014) and sympathetic nerve activity (Heffernan et al. 2007), alongside increases in nitric-oxide bioavailability and endothelial function (Campbell et al. 2011). Elevations in arterial stiffness following exercise in obese and hypertensive adults (Shim et al. 2011, Gkaliagkousi et al. 2014, Moon et al. 2015, Bunsawat et al. 2017) are potentially detrimental, and may be due to underlying 
dysfunction of these mechanisms. Indeed, there is evidence of altered sympathetic baroreflex sensitivity that is exacerbated with exercise (Okada et al. 2013), revealing underlying dysfunction that is not evident at rest (Shim et al. 2011). As wave reflection characteristics are reported to represent reflected pressure waves from peripheral arteries and are influenced by changes in downstream artery function, it is likely that the decreases we observed in wave reflection (AIx75 and reflection magnitude) following exercise, in contrast to the negligible changes in PWV, are primarily due to peripheral vasodilation and the reduced vascular resistance within the exercising limbs.

\section{Reliability of arterial stiffness indices during exercise recovery}

The reliability of post-exercise recovery measures of arterial stiffness was similar to that observed at rest. Reliability of the recovery of arterial stiffness following exercise was excellent for PWV and AIx 75, but poor for RM despite good reliability of its determinants (Pf: ICC 0.68, Pb: ICC 0.75). Our results for AIx 75 were in agreement with a recent study in younger adults that assessed the reliability of the changes in AIx75 during 30 min of recovery after incremental cycling exercise (ICC: 0.82 ) (Lim et al. 2016). An earlier return of the reflected wave has been observed in older adults (Namasivayam et al. 2009), and likely causes the movement of the inflection point (the point where the forward and backward wave meet (Westerhof et al. 2006)) into late systole that makes these indices less distinguishable (O'Rourke et al. 2005, Westerhof et al. 2012). In addition, our assessment of the AUC of the complete recovery period likely increases the variability of this index compared to measurements at a single time point. In combination with the low ICC values for RM at rest, RM may not be sensitive to meaningful post-exercise changes in older individuals. We show that the postexercise response of PWV and AIx75, but not RM, have strong reliability in older adults.

The clinical significance of changes in arterial stiffness after exercise is not yet established, and this study provides the basis for future research in this area. Given the excellent reliability of the PWV response to exercise in older adults, the utility of this measurement as a prognostic marker should be considered. The reliability coefficient, or smallest detectable change, in the immediate response of 
PWV after exercise $\left(1.9 \mathrm{~m} . \mathrm{s}^{-1}\right)$ was similar to the change in PWV previously observed after exercise (increase of $\sim 2.0 \mathrm{~m} \cdot \mathrm{s}^{-1}$ ) in adults with untreated grade I hypertension (Gkaliagkousi et al. 2014). Conversely, an increase in PWV of $1.3 \mathrm{~m} . \mathrm{s}^{-1}$ following exercise has been associated with abnormal kidney function in middle-aged adults (Keith et al. 2013), however our data suggests that this is below the smallest detectable change in older adults. For future studies exploring the utility of the immediate change in PWV following exercise, we estimate that a cohort of 8 (assuming a sample SD of $1 \mathrm{~m} \cdot \mathrm{s}^{-1}$ ) to 17 (SD of $2 \mathrm{~m} \cdot \mathrm{s}^{-1}$ ) participants per group (with $>80 \%$ power) would be required to reveal significant differences that correspond with the smallest detectable change $\left(1.9{\mathrm{~m} . \mathrm{s}^{-1}}^{-1}\right)$ in the PWV response.

A strength of this study is that we report the between-day reliability of resting and post-exercise arterial stiffness indices in older healthy individuals, using an observer-independent portable device. Our study also included males and females within the target age-range, which helps to generalise our findings to older healthy adults. There are also some limitations. Our results are not necessarily transferable to older individuals with cardiovascular disease, for which these measurements would be of particular interest. Furthermore, future studies may wish to confirm our findings using highintensity or maximal exercise as this is associated with the greatest exercise-induced risk (Rognmo et al. 2012). Nonetheless, moderate-intensity exercise, as used in this study, is recommended for olderadults in line with current exercise guidelines for health in older adults (Garber et al. 2011).

\section{Conclusion}

This study established the reliability of arterial stiffness indices at rest, immediately after moderateintensity cycling exercise and during supine recovery in healthy older adults. We demonstrated that post-exercise measures of arterial stiffness are as reliable as measurements under resting conditions. These findings provide new information about the smallest detectable changes in arterial stiffness with exercise, and the potential use of these measures for the assessment of cardiovascular risk. Further investigation of the physiological determinants and clinical significance of post-exercise measures of arterial stiffness is warranted. 


\section{Acknowledgements}

This research was supported by grants from the National Health and Medical Research Council (1000967, 1022752, 1079369), The Townsville Hospital and the Inflammation and Healing Research Cluster at the University of the Sunshine Coast. Professor Jonathan Golledge's work is supported by fellowships from the NHMRC (1117061) and the Queensland Government (Senior Clinical Research Fellowship).

\section{Conflicts of interest.}

The authors declare no conflict of interest. 


\section{References}

Bunsawat, K., S. M. Ranadive, A. D. Lane-Cordova, H. Yan, R. M. Kappus, B. Fernhall and T. Baynard (2017). "The effect of acute maximal exercise on postexercise hemodynamics and central arterial stiffness in obese and normal-weight individuals." $\underline{\text { Physiological Reports }}$ 5(7).

Butlin, M., A. Qasem and A. P. Avolio (2012). "Estimation of central aortic pressure waveform features derived from the brachial cuff volume displacement waveform." Conf Proc IEEE Eng Med Biol Soc 2012: 2591-2594.

Campbell, R., J. P. Fisher, J. E. Sharman, B. J. McDonnell and M. P. Frenneaux (2011). "Contribution of nitric oxide to the blood pressure and arterial responses to exercise in humans." Journal of Human Hypertension 25(4): 262-270.

Cecelja, M. and P. Chowienczyk (2012). "Role of arterial stiffness in cardiovascular disease." JRSM Cardiovascular Disease 1(4): cvd.2012.012016.

Chirinos, J. A., J. G. Kips, D. R. Jacobs, Jr., L. Brumback, D. A. Duprez, R. Kronmal, D. A. Bluemke, R. R. Townsend, S. Vermeersch and P. Segers (2012). "Arterial wave reflections and incident cardiovascular events and heart failure: MESA (Multiethnic Study of Atherosclerosis)." J Am Coll Cardiol 60(21): 2170-2177.

Dhalla, N. S., R. M. Temsah and T. Netticadan (2000). "Role of oxidative stress in cardiovascular diseases." J Hypertens 18(6): 655-673.

Dhaun, N., R. Moorhouse, I. M. MacIntyre, V. Melville, W. Oosthuyzen, R. A. Kimmitt, K. E. Brown, E. D. Kennedy, J. Goddard and D. J. Webb (2014). "Diurnal Variation in Blood Pressure and Arterial Stiffness in Chronic Kidney Disease: The Role of Endothelin-1." Hypertension 64(2): 296-304.

Duprez, D. A. (2010). "Arterial Stiffness and Endothelial Function." Key Players in Vascular Health 55(3): 612-613.

Ferguson, B. (2014). "ACSM's Guidelines for Exercise Testing and Prescription 9th Ed. 2014." The Journal of the Canadian Chiropractic Association 58(3): 328-328.

Fleiss, J. L. (1999). Reliability of Measurement. The Design and Analysis of Clinical Experiments, John Wiley \& Sons, Inc.: 1-32.

Garber, C. E., B. Blissmer, M. R. Deschenes, B. A. Franklin, M. J. Lamonte, I. M. Lee, D. C. Nieman and D. P. Swain (2011). "American College of Sports Medicine position stand. Quantity and quality of exercise for developing and maintaining cardiorespiratory, musculoskeletal, and neuromotor fitness in apparently healthy adults: guidance for prescribing exercise." Med Sci Sports Exerc 43(7): 1334-1359. 
Gkaliagkousi, E., E. Gavriilaki, B. Nikolaidou, G. Triantafyllou and S. Douma (2014). "ExerciseInduced Pulse Wave Velocity Changes in Untreated Patients With Essential Hypertension: The Effect of an Angiotensin Receptor Antagonist." The Journal of Clinical Hypertension 16(7): 482-487.

Hamilton, P. K., C. J. Lockhart, C. E. Quinn and G. E. McVeigh (2007). "Arterial stiffness: clinical relevance, measurement and treatment." Clin Sci (Lond) 113(4): 157-170.

Heffernan, K. S., S. R. Collier, E. E. Kelly, S. Y. Jae and B. Fernhall (2007). "Arterial stiffness and baroreflex sensitivity following bouts of aerobic and resistance exercise." International Journal of Sports Medicine 28(3): 197-203.

Hwang, M. H., J. K. Yoo, H. K. Kim, C. L. Hwang, K. Mackay, O. Hemstreet, W. W. Nichols and D. D. Christou (2014). "Validity and reliability of aortic pulse wave velocity and augmentation index determined by the new cuff-based SphygmoCor Xcel." J Hum Hypertens 28(8): 475-481.

Kawamoto, R., D. Ninomiyax, T. Kusunoki, Y. Kasai, N. Ohtsuka and T. Kumagi (2016). "Oxidative stress is associated with increased arterial stiffness in middle-aged and elderly community-dwelling persons." Journal of Clinical Gerontology and Geriatrics 7(4): 136140.

Keith, L. J., S. Rattigan, M. A. Keske, M. Jose and J. E. Sharman (2013). "Exercise aortic stiffness: Reproducibility and relation to end-organ damage in men." Journal of Human Hypertension 27(8): 516-522.

La Rovere, M. T. and J. H. Christensen (2015). "The autonomic nervous system and cardiovascular disease: role of n-3 PUFAs." Vascul Pharmacol 71: 1-10.

Lee, H. Y. and B. H. Oh (2010). "Aging and arterial stiffness." Circulation Journal 74(11): 22572262.

Lee Stoner, J. M. Y., and Simon Fryer4 (2012). "Assessments of Arterial Stiffness and Endothelial Function Using Pulse Wave Analysis." International Journal of Vascular Medicine 2012: 9.

Lim, W., J. Faulkner, D. Lambrick and L. Stoner (2016). "Reliability of oscillometric central blood pressure responses to submaximal exercise." J Hypertens 34(6): 1084-1090.

McDermott, M. M., K. Liu, J. M. Guralnik, S. Mehta, M. H. Criqui, G. J. Martin and P. Greenland (1998). "The ankle brachial index independently predicts walking velocity and walking endurance in peripheral arterial disease." J Am Geriatr Soc 46(11): 1355-1362.

McEniery, C. M., Yasmin, I. R. Hall, A. Qasem, I. B. Wilkinson, J. R. Cockcroft and A. Investigators (2005). "Normal vascular aging: differential effects on wave reflection and aortic pulse wave velocity: the Anglo-Cardiff Collaborative Trial (ACCT)." J Am Coll Cardiol 46(9): 1753-1760. 
Millen, A. M., A. J. Woodiwiss and G. R. Norton (2016). "Post-exercise effects on aortic wave reflection derived from wave separation analysis in young- to middle-aged prehypertensives and hypertensives." Eur J Appl Physiol.

Mitchell, G. F., H. Parise, E. J. Benjamin, M. G. Larson, M. J. Keyes, J. A. Vita, R. S. Vasan and D. Levy (2004). "Changes in arterial stiffness and wave reflection with advancing age in healthy men and women: the Framingham Heart Study." Hypertension 43(6): 1239-1245.

Mitchell, G. F., S.-J. Hwang, R. S. Vasan, M. G. Larson, M. J. Pencina, N. M. Hamburg, J. A. Vita, D. Levy and E. J. Benjamin (2010). "Arterial Stiffness and Cardiovascular Events: The Framingham Heart Study." Circulation 121(4): 505-511.

Mitchelmore, A., L. Stoner, D. Lambrick, S. Jobson and J. Faulkner (2017). "Reliability of oscillometric central blood pressure and central systolic loading in individuals over 50 years: Effects of posture and fasting." Atherosclerosis 269: 79-85.

Moon, S.-H., J.-C. Moon, D.-H. Heo, Y.-H. Lim, J.-H. Choi, S.-Y. Kim, K.-S. Kim and S.-J. Joo (2015). "Increased pulse wave velocity and augmentation index after isometric handgrip exercise in patients with coronary artery disease." Clinical Hypertension 21: 5.

Mutter, A. F., A. B. Cooke, O. Saleh, Y. H. Gomez and S. S. Daskalopoulou (2017). "A systematic review on the effect of acute aerobic exercise on arterial stiffness reveals a differential response in the upper and lower arterial segments." Hypertension Research 40(2): 146-172.

Namasivayam, M., B. J. McDonnell, C. M. McEniery and M. F. O'Rourke (2009). "Does wave reflection dominate age-related change in aortic blood pressure across the human life span?" Hypertension 53(6): 979-985.

Nichols, W. W. and B. M. Singh (2002). "Augmentation index as a measure of peripheral vascular disease state." Curr Opin Cardiol 17(5): 543-551.

O’Rourke, M. F. and W. W. Nichols (2005). "Aortic Diameter, Aortic Stiffness, and Wave Reflection Increase With Age and Isolated Systolic Hypertension." Hypertension 45(4): 652-658.

Okada, Y., M. M. Galbreath, S. Shibata, S. S. Jarvis, T. B. Bivens, W. Vongpatanasin, B. D. Levine and Q. Fu (2013). "Morning blood pressure surge is associated with arterial stiffness and sympathetic baroreflex sensitivity in hypertensive seniors." Am J Physiol Heart Circ Physiol 305(6): H793-802.

Pfeiffer, K. A., J. M. Pivarnik, C. J. Womack, M. J. Reeves and R. M. Malina (2002). "Reliability and validity of the Borg and OMNI rating of perceived exertion scales in adolescent girls." Med Sci Sports Exerc 34(12): 2057-2061. 
Rognmo, Ø., T. Moholdt, H. Bakken, T. Hole, P. Mølstad, N. E. Myhr, J. Grimsmo and U. Wisløff (2012). "Cardiovascular Risk of High- Versus Moderate-Intensity Aerobic Exercise in Coronary Heart Disease Patients." Circulation.

Schreuder, T. H., J. H. van Lotringen, M. T. Hopman and D. H. Thijssen (2014). "Impact of endothelin blockade on acute exercise-induced changes in blood flow and endothelial function in type 2 diabetes mellitus." Exp Physiol 99(9): 1253-1264.

Schultz, M. G., A. La Gerche and J. E. Sharman (2017). "Blood Pressure Response to Exercise and Cardiovascular Disease." Curr Hypertens Rep 19(11): 89.

Shim, C. Y., W. I. Yang, S. Park, M. K. Kang, Y. G. Ko, D. Choi, Y. Jang, N. Chung and J. W. Ha (2011). "Overweight and its association with aortic pressure wave reflection after exercise." American Journal of Hypertension 24(10): 1136-1142.

Smith, M. M., C. A. Buffington, R. L. Hamlin and S. T. Devor (2015). "Relationship between muscle sympathetic nerve activity and aortic wave reflection characteristics in aerobic- and resistance-trained subjects." Eur J Appl Physiol 115(12): 2609-2619.

Steppan, J., V. Barodka, D. E. Berkowitz and D. Nyhan (2011). "Vascular Stiffness and Increased Pulse Pressure in the Aging Cardiovascular System." Cardiology Research and Practice 2011: 8 .

Stoner, L., D. Credeur, S. Fryer, J. Faulkner, D. Lambrick and B. B. Gibbs (2016). "Reliability of pulse waveform separation analysis: effects of posture and fasting." J Hypertens.

Tallarida, R. J. and R. B. Murray (1987). Area under a Curve: Trapezoidal and Simpson's Rules. Manual of Pharmacologic Calculations: With Computer Programs. New York, NY, Springer New York: 77-81.

Vaz, S., T. Falkmer, A. E. Passmore, R. Parsons and P. Andreou (2013). "The Case for Using the Repeatability Coefficient When Calculating Test-Retest Reliability." PLoS ONE 8(9): e73990.

Vlachopoulos, C., K. Aznaouridis and C. Stefanadis (2010). "Prediction of cardiovascular events and all-cause mortality with arterial stiffness: a systematic review and meta-analysis." $\mathrm{J}$ Am Coll Cardiol 55(13): 1318-1327.

Weir, J. P. (2005). "Quantifying test-retest reliability using the intraclass correlation coefficient and the SEM." J Strength Cond Res 19(1): 231-240.

Westerhof, B. E. and N. Westerhof (2012). "Magnitude and return time of the reflected wave: the effects of large artery stiffness and aortic geometry." J Hypertens 30(5): 932-939.

Westerhof, B. E., I. Guelen, N. Westerhof, J. M. Karemaker and A. Avolio (2006). "Quantification of Wave Reflection in the Human Aorta From Pressure Alone: A Proof of Principle." Hypertension 48(4): 595-601. 
Widmer, R. J. and A. Lerman (2014). "Endothelial dysfunction and cardiovascular disease." Global Cardiology Science \& Practice 2014(3): 291-308.

Wilkinson, I. B., C. M. McEniery, G. Schillaci, P. Boutouyrie, P. Segers, A. Donald and P. J. Chowienczyk (2010). "ARTERY Society guidelines for validation of non-invasive haemodynamic measurement devices: Part 1, arterial pulse wave velocity." Artery Research 4(2): 34-40. 


\section{Tables and captions}

Table 1. Participant characteristics and cardiorespiratory fitness.

BMI: body mass index; BP: blood pressure; $\mathrm{VO}_{2 \text { peak: }}$ Peak oxygen consumption.

\begin{tabular}{|c|c|}
\hline Variable & Mean \pm SD \\
\hline Age, years & $71 \pm 5$ \\
\hline Male, \% & 70 \\
\hline Weight, kg & $70 \pm 12$ \\
\hline Height, cm & $173 \pm 11$ \\
\hline BMI, kg. m² & $24 \pm 3$ \\
\hline Heart rate, bpm & $59 \pm 6.5$ \\
\hline Systolic BP, mmHg & $130 \pm 16$ \\
\hline Diastolic BP, mmHg & $75 \pm 9$ \\
\hline \multicolumn{2}{|c|}{ Maximal incremental cycling test } \\
\hline $\mathrm{VO}_{2 \text { peak }}, \mathrm{ml} \cdot \mathrm{kg}^{-1} \cdot \mathrm{min}^{-1}$ & $24.6 \pm 8.0$ \\
\hline Peak power output, $\mathrm{W}$ & $140 \pm 51$ \\
\hline Peak heart rate, bpm & $144 \pm 19$ \\
\hline
\end{tabular}


Table 2. Change of PWV, Alx75 and RM from baseline at 0, 20, 40 and 60 min post exercise.

Alx75, augmentation index normalised to a heart rate of $75 \mathrm{bpm}$; RM, reflexion magnitude; PWV, pulse wave velocity, $\Delta$ denotes delta (change).

\begin{tabular}{|c|c|c|c|c|c|c|}
\hline Variable & Visit & 0 min post & 20 min post & 40 min post & 60 min post & P-Value \\
\hline \multirow{3}{*}{$\Delta \mathbf{P W V}\left(\mathbf{m} . \mathbf{s}^{-1}\right)$} & Visit 1 & $0.9 \pm 1.0$ & $0.4 \pm 0.6$ & $0.5 \pm 0.7$ & $0.6 \pm 0.8$ & Visit :0.241 \\
\hline & Visit 2 & $0.5 \pm 0.8$ & $0.2 \pm 0.7$ & $0.2 \pm 1.0$ & $0.4 \pm 1.2$ & Time:0.019 \\
\hline & Visit 3 & $0.4 \pm 0.4$ & $0.3 \pm 0.8$ & $0.4 \pm 0.8$ & $0.4 \pm 0.9$ & Visit*time:0.398 \\
\hline \multirow{3}{*}{$\Delta \mathrm{AIx} @ 75$ (\%) } & Visit 1 & $-0.1 \pm 9$ & $2.6 \pm 7$ & $-2.5 \pm 5.2$ & $-3.6 \pm 4$ & Visit :0.851 \\
\hline & Visit 2 & $2.4 \pm 4$ & $1.7 \pm 2$ & $-2.9 \pm 4$ & $-3.6 \pm 4$ & Time:0.001 \\
\hline & Visit 3 & $1.0 \pm 6$ & $1.2 \pm 4.6$ & $-3.7 \pm 3$ & $-3.4 \pm 5$ & Visit*time: 0.943 \\
\hline \multirow{3}{*}{$\Delta \mathbf{R M}(\%)$} & Visit 1 & $-14.9 \pm 15$ & $-3.4 \pm 15$ & $-6.1 \pm 10$ & $-0.1 \pm 13$ & Visit: 0.211 \\
\hline & Visit 2 & $-9.1 \pm 10$ & $-9.4 \pm 10$ & $-3.1 \pm 10$ & $-3.3 \pm 11$ & Time: 0.001 \\
\hline & Visit 3 & $-6.1 \pm 8$ & $-2.9 \pm 9$ & $1.7 \pm 12$ & $-3.4 \pm 10$ & Visit*time: 0.398 \\
\hline
\end{tabular}


Table 3. Reliability of arterial stiffness indices at rest, immediately post exercise and during exercise recovery.

$\mathrm{P}<0.05$ indicates significant difference between visits; data are presented as mean $\pm \mathrm{SD}$; ICC, intraclass correlation; Immediately post (0 min); CV\%, coefficient of variation; SEM, standard error of the mean; RC, reliability coefficient; Alx75, augmentation index normalised to a heart rate of $75 \mathrm{bpm}$; Pf, forward pressure wave; $\mathrm{Pb}$, backward pressure wave; RM, reflexion magnitude; PWV, pulse wave velocity; HR, heart rate; SBP, systolic blood pressure; DBP diastolic blood pressure.

\begin{tabular}{|c|c|c|c|c|c|c|c|c|c|}
\hline Variable & & Visit 1 & Visit 2 & Visit 3 & $I C C$ & $C V \%$ & SEM & $R C$ & P-value \\
\hline \multirow{3}{*}{ PWV (m.s $\left.{ }^{-1}\right)$} & Rest & $11.2 \pm 2.4$ & $11.2 \pm 2.1$ & $10.7 \pm 2$ & 0.91 & 5.0 & 0.7 & 1.9 & 0.84 \\
\hline & Immediately post & $12.2 \pm 2$ & $11.79 \pm 2.4$ & $11.31 \pm 2$ & 0.87 & 6.5 & 0.7 & 1.9 & 0.26 \\
\hline & Recovery $(A U C)$ & $721 \pm 150$ & $692 \pm 133$ & $677 \pm 129$ & 0.94 & 4.9 & 56 & 154 & 0.78 \\
\hline \multirow{3}{*}{ Alx@75 (\%) } & Rest & $25 \pm 9$ & $22 \pm 8$ & $23 \pm 8$ & 0.72 & 14.0 & 3 & 7 & 0.24 \\
\hline & Immediately post & $25 \pm 10$ & $24 \pm 8$ & $24 \pm 5$ & 0.67 & 16.7 & 2 & 7 & 0.99 \\
\hline & Recovery $(A U C)$ & $1441 \pm 365$ & $1284 \pm 416$ & $1330 \pm 400$ & 0.84 & 11.7 & 166 & 462 & 0.68 \\
\hline \multirow{3}{*}{ Pf (mmHg) } & Rest & $24.5 \pm 7$ & $23.7 \pm 6$ & $23.3 \pm 5$ & 0.71 & 9.4 & 1.8 & 4.8 & 0.12 \\
\hline & Immediately post & $31.2 \pm 8$ & $28.4 \pm 6$ & $27.3 \pm 6$ & 0.54 & 13.2 & 2.0 & 5.6 & 0.38 \\
\hline & Recovery $(A U C)$ & $1586 \pm 276$ & $1570 \pm 311$ & $1414 \pm 349$ & 0.68 & 8.9 & 131 & 364 & 0.44 \\
\hline \multirow{3}{*}{$\mathrm{Pb}$ (mmHg) } & Rest & $18.2 \pm 3$ & $17.4 \pm 2$ & $17.8 \pm 3$ & 0.54 & 9.0 & 0.8 & 2.2 & 0.34 \\
\hline & Immediately post & $19.4 \pm 4$ & $18.9 \pm 4$ & $19.5 \pm 5$ & 0.73 & 10.5 & 1.3 & 3.6 & 0.94 \\
\hline & Recovery (AUC) & $1093 \pm 187$ & $1072 \pm 141$ & $1052 \pm 242$ & 0.75 & 7.6 & 73 & 202 & 0.90 \\
\hline \multirow{3}{*}{ RM (\%) } & Rest & $75.5 \pm 13$ & $74.6 \pm 13$ & $74.5 \pm 11$ & 0.40 & 11.5 & 4 & 11 & 0.98 \\
\hline & Immediately post & $61 \pm 11$ & $66 \pm 11$ & $68 \pm 10$ & 0.59 & 9.8 & 3 & 9 & 0.26 \\
\hline & Recovery $(A U C)$ & $4190 \pm 432$ & $4102 \pm 532$ & $4195 \pm 399$ & 0.38 & 9.1 & 180 & 500 & 0.89 \\
\hline \multirow{3}{*}{ HR (b.min $\left.{ }^{-1}\right)$} & Rest & $60 \pm 8$ & $60 \pm 8$ & $59 \pm 7$ & 0.70 & 4.9 & 3 & 7 & 0.78 \\
\hline & Immediately post & $70 \pm 8$ & $69 \pm 10$ & $68 \pm 10$ & 0.62 & 5.7 & 3 & 8 & 0.81 \\
\hline & Recovery $(A U C)$ & $534 \pm 170$ & $590 \pm 190$ & $580 \pm 185$ & 0.77 & 4.8 & 170 & 502 & 0.62 \\
\hline \multirow{3}{*}{$\begin{array}{c}\text { SBP } \\
(\mathrm{mmHg})\end{array}$} & Rest & $127 \pm 18$ & $120 \pm 13$ & $118 \pm 12$ & 0.80 & 5.0 & 5 & 12 & 0.78 \\
\hline & Immediately post & $134 \pm 17$ & $130 \pm 18$ & $128 \pm 13$ & 0.81 & 5.8 & 5 & 14 & 0.71 \\
\hline & Recovery (AUC) & $10220 \pm 1250$ & $10025 \pm 1180$ & $9800 \pm 930$ & 0.85 & 3.5 & 355 & 984 & 0.65 \\
\hline \multirow{3}{*}{ DBP(mmHg) } & Rest & $75 \pm 13$ & $70 \pm 9$ & $71 \pm 10$ & 0.76 & 5.3 & 3 & 9 & 0.53 \\
\hline & Immediately post & $78 \pm 9$ & $76 \pm 12$ & $75 \pm 10$ & 0.89 & 4.7 & 3 & 8 & 0.64 \\
\hline & Recovery $(A U C)$ & $6090 \pm 780$ & $5900 \pm 780$ & $5740 \pm 730$ & 0.91 & 3.5 & 242 & 650 & 0.57 \\
\hline
\end{tabular}



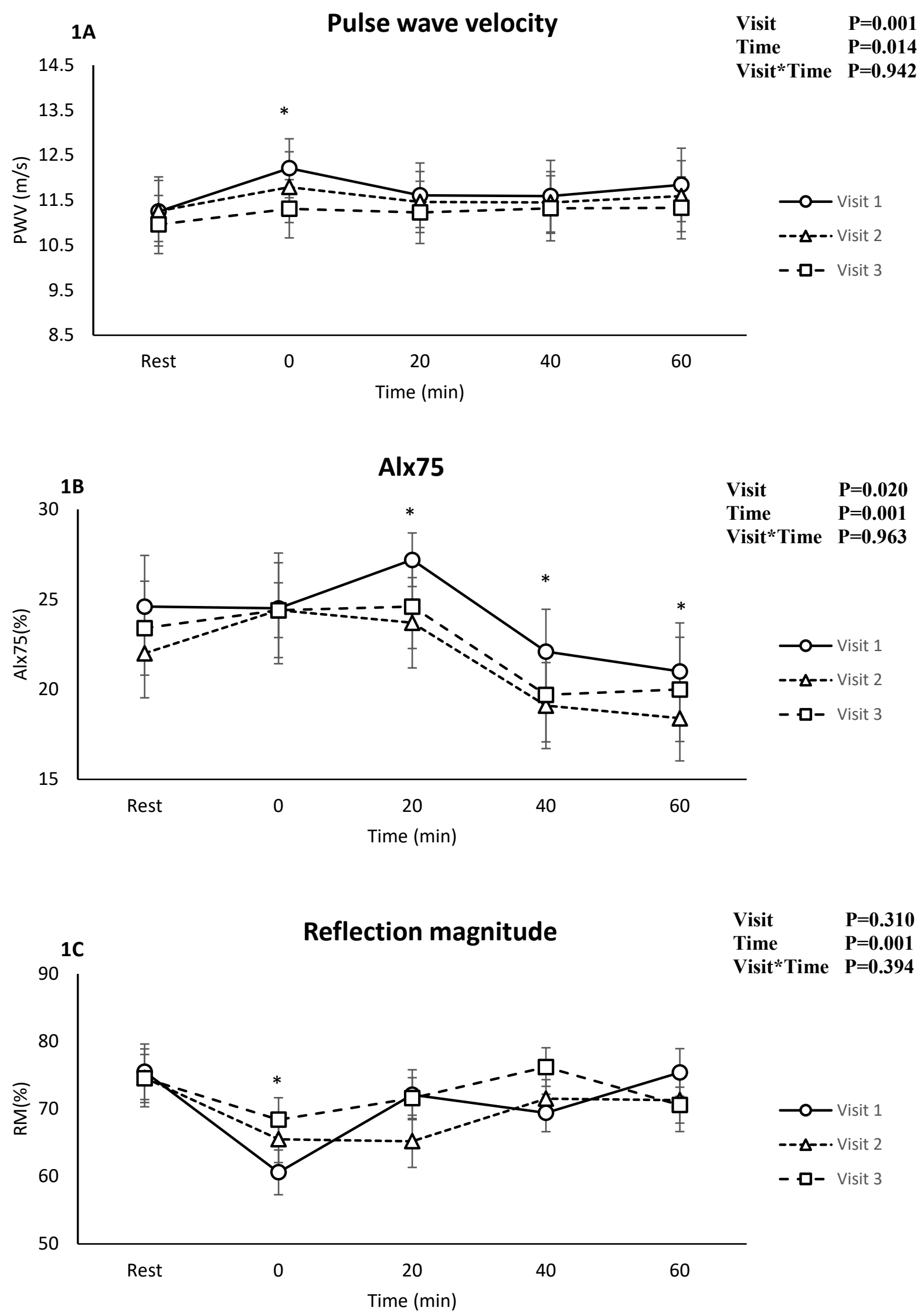
Figure 1. PWV (A), Alx75 (B) and RM (C) at rest and post-exercise during visit 1 (white circles), visit 2 (white triangles) and visit 3 (white squares). Error bars represent SD. *indicates significant change across time compared to baseline; post hoc analysis of visit effect revealed mean PWV was lower during visit 3 compared to visit $1(\mathrm{p}<0.001)$ and $2(\mathrm{p}=0.042)$, and mean AIx75 was higher in visit 1 compared to visit $2(\mathrm{p}=0.006)$. 